\title{
Fringe-field effects on the time evolution of pendular states
}

\author{
Rafael Escribano, Belén Maté, Félix Ortigoso, and Juan Ortigoso \\ Instituto de Estructura de la Materia, Consejo Superior de Investigaciones Científicas, Serrano 121-123, 28006 Madrid, Spain
}

(Received 23 February 2000; published 20 July 2000)

\begin{abstract}
The interaction of the permanent electric dipole moment of a polar molecule with a strong static electric field can create oriented rotational wave packets, which are termed pendular states. Molecules traveling in a molecular beam experience a fringing field due to the edges of the conducting plates which create the electric field as a slowly varying time-dependent perturbation. Since Stark energies of some asymmetric-top molecules present numerous avoided crossings as a function of field strength, the time evolution of rotational wave functions for molecules entering the field is complex. We have studied, by solving the time-dependent Schrödinger equation, the dynamics caused by selected avoided crossings, and the time evolution of the orientation corresponding to 24 rotational wave functions, for the near-prolate asymmetric-top iodobenzene. Implications for the population distribution of molecules in the electric field and for experimental schemes to decelerate molecules are discussed.
\end{abstract}

PACS number(s): 33.55.Be, 33.80.-b, 03.65.Ge, 33.90.+h

\section{INTRODUCTION}

The study of the interaction of molecules with strong electric fields is becoming a topic of great interest in chemical physics. The possibility of orienting polar molecules by combining cooled molecular beams with strong static electric fields was studied by Loesch and Remscheid [1] and Friedrich and Herschbach [2]. Also, ensembles of nonpolar molecules can be aligned (but not oriented) by using intense pulsed lasers, which interact with the molecular polarizability, inducing a dipole moment. The induced dipole can further interact with the laser field, giving rise to pendular states [3]. These states are field-induced coherent superpositions of field-free rotor states. For high enough field strengths, molecules, in these hybrid states, librate about the field direction instead of executing free rotational motion. The time evolution of pendular states created by nonresonant lasers was recently studied for linear molecules [4], to determine the character (adiabatic versus diabatic) of the evolution from field-free eigenstates to aligned states. A variety of behaviors was found depending on the duration and intensity of the laser pulse and the rotational constant of the molecule.

Research on pendular states has concentrated mainly on linear and symmetric-top molecules but also studies on asymmetric tops have been published. Moore et al. [5] presented a comprehensive theoretical and experimental study of the spectroscopy of acetylene-HF (a near-prolate asymmetric top), identifying the molecular states that give the best orientation in a static electric field. Recently, Larsen et al. [6] have demonstrated laser-induced alignment for linear, symmetric-top and asymmetric-top molecules. These authors also discussed some interesting applications of aligned molecules, like dissociative rotational cooling, selective dissociation, and chemical control.

Moore et al. [5] finished their paper commenting on the issue of nonadiabatic population transfer that can take place when molecules enter the electric field. This issue is important for determining the relative rotational populations inside the field. If the time evolution at an avoided crossing (AC) is completely adiabatic or completely diabatic a molecule will be described at any time by only one molecule-field eigenstate (a Stark eigenstate in the case of dc fields or a Floquet state in the case of ac fields), whose relative population will be the same as its field-free progenitor. Intermediate behavior between fully adiabatic or fully diabatic evolution is possible, implying that molecules end up in a coherent superposition of molecule-field eigenstates. As Bulthuis et al. [7] pointed out "a calculation assuming [that the] avoided crossings (are transversed adiabatically) can be expected to lead to completely incorrect orientation distributions for the various parent states in a thermal distribution (of iodobenzene)."

The usual experimental configuration to create pendular states consists of a well-defined molecular beam passing through a couple of electrodes, which create a dc electric field. The transition from the field-free region to the highfield region does not occur suddenly due to the edge effect of the conducting plates. This fringe electric field is experienced by the molecules as a slowly varying time-dependent perturbation, whose effective variation depends on the molecular velocity. Since the energy level structure of asymmetric-top molecules is more complex than that of linear or symmetric-top molecules, the existence of avoided crossings as a function of the electric-field strength occurs often [7]. Molecules transverse avoided crossings fully adiabatically, fully diabatically, or in an intermediate fashion, depending on how fast the field changes compared to the energy gap between the two Stark states involved in the crossing.

In this paper, we present detailed calculations of the time evolution of rotational wave functions for the near-prolate asymmetric-top iodobenzene, from the field-free region to the static-field region. For typical molecular velocities and moderately strong electric fields the time-dependent Schrödinger equation has to be integrated for an extremely long time (several microseconds), which can be efficiently done thanks to a transformation proposed in Ref. [8] combined with the split-operator technique [9]. We have chosen iodobenzene since its Stark eigenstates have been carefully studied [7], and laser-induced alignment has been recently demonstrated for this molecule [6]. Iodobenzene represents a 
difficult problem because of its weak asymmetry, which gives rise to a large number of interacting states and avoided crossings. More asymmetric molecules, like water, do not show curve crossings [7] and the time evolution is adiabatic.

The variation of the electric-field strength as a function of space-fixed coordinates for an ideal experimental configuration, the Hamiltonian, and the algorithm used to integrate the time-dependent Schrödinger equation are given in Sec. II. Results for iodobenzene are presented and analyzed in Sec. III. Finally, in Sec. IV, we summarize and discuss our results.

\section{HAMILTONIAN, ELECTRIC FIELD, AND TIME- DEPENDENT SCHRÖDINGER EQUATION}

The Hamiltonian describing the Stark energy levels and wave functions of an asymmetric-top molecule in an isolated vibronic state (assumed for simplicity the ground state), in the presence of an electric field $\varepsilon(t)$ linearly polarized along the space-fixed direction $Z$, in the dipole approximation is

$$
H(t)=A J_{z}^{2}+B J_{x}^{2}+C J_{y}^{2}-\mu_{Z} \varepsilon(t),
$$

where $A, B$, and $C$ are rotational constants, and $J_{\gamma}$ is the component of the angular momentum operator along the molecule-fixed principal axis of inertia, $\gamma$. The component of the permanent dipole moment of the molecule along the laboratory-fixed field direction $\mu_{Z}$ can be expanded as a function of the molecule-fixed dipole components, using direction cosines $\lambda_{\Gamma \gamma}$ as

$$
\mu_{Z}=\mu_{x} \lambda_{Z x}+\mu_{y} \lambda_{Z y}+\mu_{z} \lambda_{Z z}
$$

The calculation of matrix elements can be simplified by using complex spherical coordinates [10]:

$$
\begin{aligned}
\mu_{Z}= & \mu_{z} \lambda_{Z z}+\left(\mu_{x}+i \mu_{y}\right)\left(\lambda_{Z x}-i \lambda_{Z y}\right) \\
& +\left(\mu_{x}-i \mu_{y}\right)\left(\lambda_{Z x}+i \lambda_{Z y}\right) .
\end{aligned}
$$

Finally, using the traditional $\mathrm{I}^{r}$ convention [11], the Hamiltonian becomes

$$
\begin{aligned}
H(t)= & A J_{a}^{2}+B J_{b}^{2}+C J_{c}^{2}-\left[\mu_{a} \lambda_{a}+\left(\mu_{b}+i \mu_{c}\right)\left(\lambda_{b}-i \lambda_{c}\right)\right. \\
& \left.+\left(\mu_{b}-i \mu_{c}\right)\left(\lambda_{b}+i \lambda_{c}\right)\right] \varepsilon(t) .
\end{aligned}
$$

Direction cosine matrix elements in a basis set of symmetric-top wave functions $|J, k, M\rangle$ (where $J$ is the angular momentum value, $k$ its projection along the moleculefixed axis $a$, and $M$ its projection along the space-fixed axis $Z)$ can be found in Ref. [12]. The direction cosine $\lambda_{a}$ gives rise to matrix elements with $\Delta k=0$, while the terms $\lambda_{b}$ $\pm i \lambda_{c}$ couple basis functions with $\Delta k= \pm 1$. For a given matrix element only one of the three terms can be nonzero. Also, only matrix elements between basis functions with $\Delta J=0, \pm 1$ are nonzero, and, for a linearly polarized field, $\Delta M=0$. Matrix elements for the components of the angular momentum operator with the phases chosen consistently respect to Ref. [12] are given in Ref. [11].

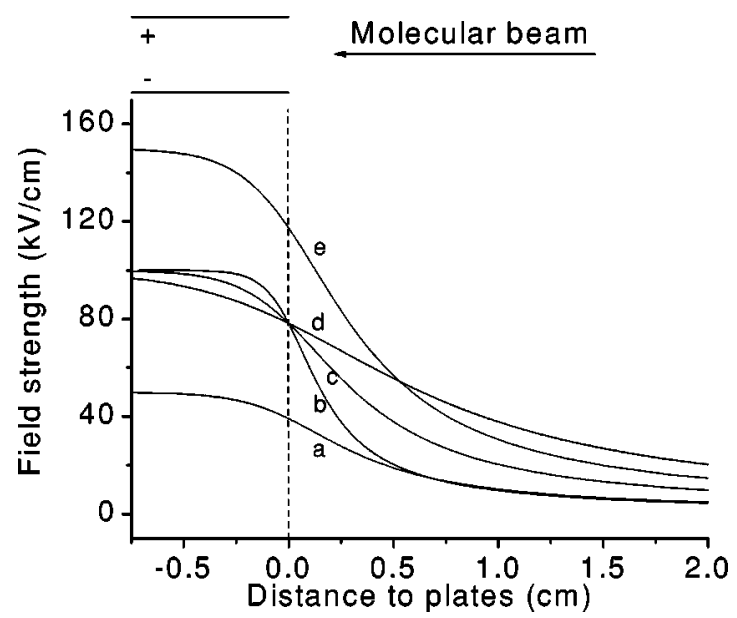

FIG. 1. Electric-field strength variation as a function of the distance to the edges of the two conducting plates that create the field. The different electric fields correspond to the following potential differences $V_{0}$ and gap between plates, $a$ : (a) $V_{0}=50 \mathrm{kV}, a$ $=1 \mathrm{~cm}$, (b) $V_{0}=50 \mathrm{kV}, a=0.5 \mathrm{~cm}$, (c) $V_{0}=100 \mathrm{kV}, a=1 \mathrm{~cm}$, (d) $V_{0}=200 \mathrm{kV}, a=2 \mathrm{~cm}$, and (e) $V_{0}=150 \mathrm{kV}, a=1 \mathrm{~cm}$.

We assume that the electric field is created by applying a potential difference between two rectangular semi-infinite conducting plates (see Fig. 1). We restrict our analysis to molecules on the center line of the midplane between the parallel plates ( $X$ axis, $Z=0, Y=0$ ), where the potential is zero. Molecules moving in that line are not defocused. The component $\varepsilon_{Z}$ at the distance $d$ is given by

$$
\left(\varepsilon_{Z}\right)_{d}^{V=0}=\left(\frac{\partial V}{\partial Z}\right)_{d}^{V=0}=\frac{\left(-V_{0} / a\right)}{1+e^{\pi / / V_{0}}},
$$

where $V_{0}$ is the difference of potential applied to the plates, $a$ is the distance between plates, and $\chi$ is the electric flux, which at the point $d$ can be determined from

$$
d=(a / 2 \pi)\left(1+\pi \chi / V_{0}+e^{\pi \chi / V_{0}}\right) .
$$

By solving Eqs. (2.5) and (2.6) the electric field experienced by a molecule traveling in the molecular beam, at time $t$, is easily determined if the molecular velocity $v$ is known. Figure 1 shows the variation of the electric-field strength as a function of the distance from the edge of the plates for different $V_{0}$ and $a$ values. The field is still nonzero far from the plate edges (experimental arrangements in which the fields are screened are possible, but we do not consider this sophistication here). For example, for $V_{0}=100 \mathrm{kV}$ and $a=1 \mathrm{~cm}$, the field is approximately $2.9 \mathrm{kV} / \mathrm{cm}$ at $6 \mathrm{~cm}$ from the plates and $20 \mathrm{kV} / \mathrm{cm}$ at $1 \mathrm{~cm}$.

The time evolution of a rotational wave function under the influence of Hamiltonian Eq. (2.4) can be calculated by solving the time-dependent Schrödinger equation. If we expand a generic rotational wave function at time $t$ as a series of eigenstates of the field-free asymmetric-top Hamiltonian $H_{\mathrm{rot}}=A J_{a}^{2}+B J_{b}^{2}+C J_{c}^{2}, \quad \psi(t)=\Sigma_{n} c_{n}(t)\left|J, K_{a}, K_{c}, M\right\rangle_{n}$ (where $K_{a}$ and $K_{c}$ indicate, respectively, the prolate symmetric-top and oblate symmetric-top levels with which 
each asymmetric-top energy level is correlated [11]) the Schrödinger equation can be written as

$$
i \frac{d}{d t} \mathbf{c}(t)=\mathbf{H}(t) \mathbf{c}(t) .
$$

The wave function at time $t_{1}$ is given by

$$
\psi\left(t_{1}\right)=U\left(t_{1}, t_{0}\right) \psi\left(t_{0}\right)
$$

the propagator can be calculated by discretizing the time interval, i.e., $U\left(t_{1}, t_{0}\right)=T \prod_{k=1}^{n} \exp \left[-i H\left(t_{k}\right) \Delta t_{k}\right]$, where $T$, the time-ordering operator that arranges the product in chronological order, appears because $H(t)$ does not commute with itself at individual times within the interval $\left[t_{0}, t_{1}\right]$. The exponential, for each subinterval, can be calculated using the split-operator technique [9]. If the time-dependent part and the coordinate-dependent part of the molecule-field interaction can be separated in independent factors, as occurs in our case, it is possible to transform the time-dependent Schrödinger equation in the following way [8]:

$$
i \frac{d}{d t} \widetilde{\mathbf{c}}(t)=\left[\mathbf{B}^{t} \mathbf{H}_{\mathrm{rot}} \mathbf{B}-\tilde{\mu}_{Z} \varepsilon(t)\right] \tilde{\mathbf{c}}(t),
$$

where $\tilde{\mu}_{Z} \equiv \mathbf{B}^{t} \mu_{Z} \mathbf{B}$ is the diagonalized effective dipolemoment matrix, and $\widetilde{\mathbf{c}}(t)=\mathbf{B}^{t} \mathbf{c}(t)$. The short-time propagator is then

$$
\begin{aligned}
\mathbf{U}(t+\Delta t, t)= & \exp \left[i \varepsilon(t) \tilde{\mu}_{Z} \Delta t / 2\right] \\
& \times\left[\mathbf{B}^{t} \exp \left(-i \mathbf{H}_{\mathrm{rot}} \Delta t\right) \mathbf{B}\right] \exp \left[i \varepsilon(t) \tilde{\mu}_{Z} \Delta t / 2\right]
\end{aligned}
$$

and therefore only one diagonalization (of the projection of the dipole moment operator along the field direction $\mu_{Z}$ $=\mu \cos \theta)$ is required during the entire procedure.

\section{CALCULATIONS FOR IODOBENZENE}

\section{A. Stark energy levels as a function of electric-field strength}

Bulthuis et al. presented in Ref. [7] detailed calculations of the variation of Stark energies for selected states of iodobenzene and water. The Hamiltonian matrix for the field-free asymmetric-top Hamiltonian can be split, for each $J$ value, in four blocks employing Wang basis functions $\left(E^{+}, E^{-}, O^{+}\right.$, and $O^{-}$) [11]. An external field mixes states corresponding to different $J$ values and besides it can mix different Wang blocks. If $\mu_{a}$ is the only nonzero component of the dipole moment, the field can mix + and - (but not $E$ and $O$ ) Wang functions, for $M \neq 0$. However, in the special case $M=0$ the infinite Stark matrix can still be factorized into four infinite blocks, greatly simplifying the calculations. Bulthuis et al. discussed the optimal strategy to calculate Stark states for iodobenzene concluding that the symmetric-top basis set is more convenient than the Wang factorization once the field is included. However, since we restrict our calculations to the case $M=0$, Wang functions are more suitable for our purposes. The nuclear quadrupole interactions can be ne-

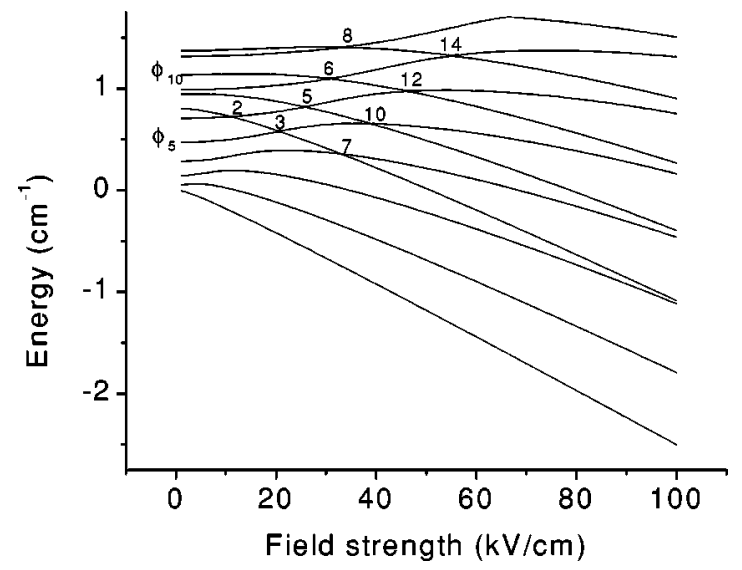

FIG. 2. Energy window for the lowest $E^{+}$Stark states with $M$ $=0\left(\phi_{1}\right.$ to $\left.\phi_{12}\right)$ for iodobenzene, as a function of electric-field strength. The avoided crossings are labeled for easy reading of Table I.

glected compared to the Stark interaction for field strengths larger than $1 \mathrm{kV} / \mathrm{cm}$, and therefore are not included in our calculations.

Rotational constants (in $\mathrm{cm}^{-1}$ ) of iodobenzene are $A$ $=0.18919, B=0.02503$, and $C=0.02210$ [13]. The dipole moment is directed along the principal axis $a$, and its value is $1.70 \mathrm{D}$ [7]. Figures 2 and 3 show the energy variation as a function of the electric field, for several $\left(M=0, E^{+}\right)$Stark eigenstates of iodobenzene up to $\varepsilon=100 \mathrm{kV} / \mathrm{cm}$. We have employed a large basis set containing the $E^{+}$Wang basis functions up to $J=25$ with $K_{a} \leqslant 8$ (the highest field-free state shown in Fig. 3 corresponds to $J=12$ ).

\section{B. Time evolution of rotational wave packets and dynamics at avoided crossings}

Bulthuis et al. [7] gave a heuristic discussion of the dynamics at avoided crossings, based on a criterion for adiabatic passage and the additional assumption that the matrix

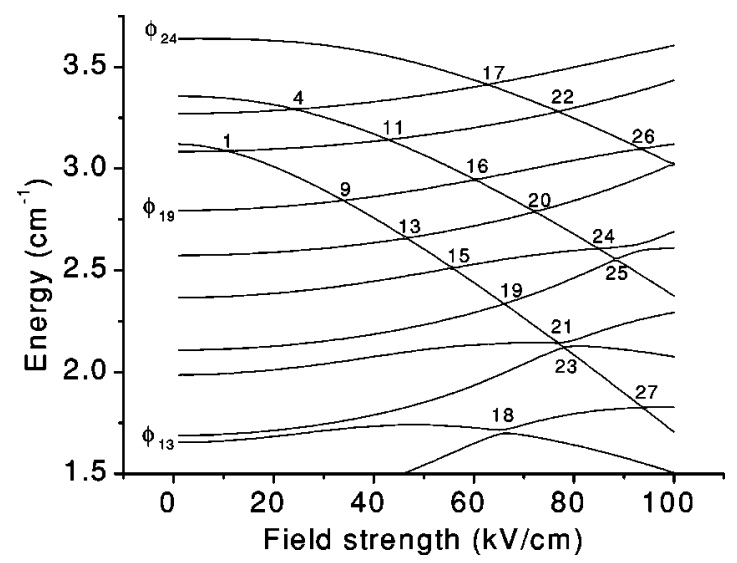

FIG. 3. Same as Fig. 2 for a different energy window. Avoided crossings are labeled for easy reading of Table I. Initial states from $\phi_{13}$ to $\phi_{24}$ are given by the following field-free eigenstates: $|6,2,4,0\rangle, \quad|8,0,8,0\rangle, \quad|7,2,5,0\rangle, \quad|9,0,9,0\rangle,|8,2,6,0\rangle,|10,0,10,0\rangle$, $|9,2,7,0\rangle, \quad|11,0,11,0\rangle, \quad|4,4,0,0\rangle, \quad|10,2,8,0\rangle, \quad|5,4,1,0\rangle, \quad$ and $|12,0,12,0\rangle$. 
elements vary linearly with the field strength. They concluded that the assumption of adiabatic crossings for a nearsymmetric top as iodobonzene is probably incorrect.

We have calculated the time evolution of the 24 lowest $\left(M=0, E^{+}\right)$rotational wave functions of iodobenzene by using the method presented in Sec. II to solve the timedependent Schrödinger equation. The Hamiltonian is given in Eq. (2.4) and the electric field corresponds to $V_{0}$ $=100 \mathrm{kV}$ and $a=1 \mathrm{~cm}$. We assume that the molecules travel in a molecular beam with mean velocity $v$ $=1600 \mathrm{~m} / \mathrm{s}$. The electrodes which create the field are considered to be placed in the free-of-collision region of the beam.

Far from the plates, as shown in Fig. 1, the field is weak and its variation is so slow that we can safely suppose that the time evolution is adiabatic before any avoided crossing takes place. Thus, for an initial wave function $\left|J, K_{a}, K_{c}, M\right\rangle$ we start the numerical propagation at $t_{i}=v / x_{i}$, where $x_{i}$ is the position for which the electric-field strength is $\varepsilon$ $=8 \mathrm{kV} / \mathrm{cm}$ (slightly smaller than the value at which the first avoided crossing takes place). Consequently, we take as initial state, the Stark state, at this field, correlating adiabatically with the chosen field-free state. Our basis set includes the same 110 basis functions used to calculate Figs. 2 and 3. The time step for the numerical integration of the Schrödinger equation was $3 \mathrm{ps}$, which gives converged results for the electric field employed.

The field-free eigenstate $\phi_{5}\left(t_{0}\right)=|4,0,4,0\rangle$ (the fifth energy level at zero field in Fig. 2) becomes, at the maximum field, a wave packet described by a linear combination of 20 asymmetric-top eigenfunctions. This apparent complexity is misleading as can be shown by projecting the wave packet $\phi\left(t_{f}\right)$ over Stark eigenfunctions $\left|\widetilde{J}, K_{a}, K_{c}, M\right\rangle$, at the final field:

$$
\phi_{5}\left(t_{f}\right)=(0.35-0.44 i)|\widetilde{4}, 0,4,0\rangle+(-0.64+0.51 i)|\widetilde{5}, 0,5,0\rangle .
$$

This result can be understood considering that AC No. 3 (near $\varepsilon=20.6 \mathrm{kV} / \mathrm{cm}$ ) is not adiabatically transversed and therefore the wave function picks up a contribution from $|\widetilde{5}, 0,5,0\rangle$ (state No. 6). However, the next crossing (AC 7) with $|\widetilde{3}, 0,3,0\rangle$ (state No. 4) is adiabatically transversed. More complicated histories are possible when the evolving wave functions pick up, in succesive avoided crossings, contributions from several instantaneous Stark states. This may be the case in highly vibrationally excited molecules where the huge density of states gives rise to a number of avoided crossings so large that eigenvalue trajectories as a function of the field get almost flat [14].

Initial states $|5,0,5,0\rangle$ (No. 6), $|2,2,0,0\rangle$ (No. 7), and $|3,2,1,0\rangle$ (No. 8) also evolve nonadiabatically:

$$
\begin{aligned}
\phi_{6}\left(t_{f}\right)= & (-0.11+0.20 i)|\widetilde{4}, 0,4,0\rangle \\
& +(-0.10+0.12 i)|\widetilde{5}, 0,5,0\rangle \\
& +(0.95-0.11 i)|\widetilde{2}, 2,0,0\rangle,
\end{aligned}
$$

$$
\begin{aligned}
\phi_{7}\left(t_{f}\right)= & (0.33-0.72|\widetilde{4}, 0,4,0\rangle+(0.34-0.42)|\widetilde{5}, 0,5,0\rangle \\
& +(0.27-0.04)|\widetilde{2}, 2,0,0\rangle, \\
\phi_{8}\left(t_{f}\right)= & 0.14|\widetilde{2}, 2,0,0\rangle+(-0.58+0.80 i)|\widetilde{3}, 2,1,0\rangle
\end{aligned}
$$

States $|0,0,0,0\rangle,|1,0,1,0\rangle$, and $|2,0,2,0\rangle$ (corresponding to the three lowest states in Fig. 2) do not exhibit avoided crossings and the numerical integration of the Schrödinger equation shows that the time evolution is adiabatic for them, as expected. States $|6,0,6,0\rangle,|4,2,2,0\rangle,|7,0,7,0\rangle$, and $|5,2,3,0\rangle$ (state Nos. 9, 10, 11, and 12 in Fig. 2) also evolve adiabatically.

The variation with the field for states $\phi_{13}$ to $\phi_{24}$ is shown in Fig. 3 (see the figure caption for the identification of these states). This energy window features 18 avoided crossings. The time propagations show that the evolved wave packets (expressed in the Stark basis set) at the final field are

$$
\begin{gathered}
\phi_{13}\left(t_{f}\right)=|\widetilde{8}, 0,8,0\rangle, \\
\phi_{14}\left(t_{f}\right)=|\widetilde{7}, 2,5,0\rangle, \\
\phi_{15}\left(t_{f}\right)=|\widetilde{9}, 0,9,0\rangle, \\
\phi_{16}\left(t_{f}\right)=|\widetilde{10}, 0,10,0\rangle, \\
\phi_{17}\left(t_{f}\right)=|\widetilde{9}, 2,7,0\rangle, \\
\phi_{18}\left(t_{f}\right)=|\widetilde{11}, 0,11,0\rangle, \\
\phi_{19}\left(t_{f}\right)=(-0.24+0.19 i)|\widetilde{4}, 4,0,0\rangle \\
+(0.83-0.47 i)|\widetilde{10}, 2,8,0\rangle, \\
\phi_{20}\left(t_{f}\right)=|\widetilde{5}, 4,1,0\rangle, \\
\phi_{21}\left(t_{f}\right)=|\widetilde{6}, 2,4,0\rangle, \\
\phi_{22}\left(t_{f}\right)=|\widetilde{12}, 0,12,0\rangle, \\
\phi_{23}\left(t_{f}\right)=|\widetilde{8}, 2,6,0\rangle, \\
\phi_{24}\left(t_{f}\right)=-0.95|\widetilde{4}, 4,0,0\rangle+0.30|\widetilde{10}, 2,8,0\rangle .
\end{gathered}
$$

These results indicate that AC Nos. 2, 3, 5, and 26 give rise to medium behavior. Crossing Nos. 6, 7, 8, 10, 12, 14, and 18 are transversed fully adiabatically. The other 16 crossings are transversed fully diabatically. Due to this variety, relative populations inside the field cannot be calculated either from field-free populations or assuming complete mixing (which implies a Bolztmann distribution based upon the energies in the field [5]).

The population of the $i$ Stark state $\left|\widetilde{J}, K_{a}, K_{c}, M\right\rangle_{i}$ at $\varepsilon\left(t_{f}\right)$ is 
TABLE I. Values for Eq. (3.19) (after multiplying by the conversion factor 0.00801586 ), and the several factors that appear in it, for the avoided crossing in Figs. 2 and 3. Indices $i$ and $j$ indicate the energy order of the Stark states involved in each AC.

\begin{tabular}{|c|c|c|c|c|c|c|c|}
\hline $\mathrm{AC}$ & $i$ & $j$ & $\varepsilon(\mathrm{kV} / \mathrm{cm})$ & $|\langle i|-\mu \cos \theta| j\rangle|$ (D) & $|\Delta E|(\mathrm{MHz})$ & $|d \varepsilon / d x|\left(\mathrm{kV} / \mathrm{cm}^{2}\right)$ & Eq. (3.19) \\
\hline 1 & 20 & 21 & 10.61758 & 0.00001 & 0.001 & 6.4 & $8.2 \times 10^{2}$ \\
\hline 2 & 6 & 7 & 10.92186 & 0.27964 & 1.992 & 6.7 & 6.1 \\
\hline 3 & 5 & 6 & 20.59211 & 0.45648 & 10.139 & 21.3 & 1.2 \\
\hline 4 & 22 & 23 & 24.23995 & 0.13275 & 0.008 & 28.1 & $7.5 \times 10^{5}$ \\
\hline 5 & 7 & 8 & 25.72210 & 0.35750 & 34.807 & 31.1 & $1.2 \times 10^{-1}$ \\
\hline 6 & 9 & 10 & 30.72066 & 0.24169 & 80.710 & 41.4 & $2.0 \times 10^{-2}$ \\
\hline 7 & 4 & 5 & 32.99955 & 0.23264 & 45.374 & 46.0 & $6.7 \times 10^{-2}$ \\
\hline 8 & 11 & 12 & 33.31518 & 0.12061 & 130.276 & 47.0 & $4.3 \times 10^{-3}$ \\
\hline 9 & 19 & 20 & 33.92624 & 0.28701 & 0.014 & 48.0 & $9.0 \times 10^{5}$ \\
\hline 10 & 6 & 7 & 38.93875 & 0.22368 & 112.114 & 58.4 & $1.3 \times 10^{-2}$ \\
\hline 11 & 21 & 22 & 42.86676 & 0.17531 & 0.002 & 66.3 & $3.7 \times 10^{7}$ \\
\hline 12 & 8 & 9 & 46.25890 & 0.21929 & 211.615 & 72.4 & $4.5 \times 10^{-3}$ \\
\hline 13 & 18 & 19 & 46.57086 & 0.30341 & 0.001 & 73.1 & $2.8 \times 10^{8}$ \\
\hline 14 & 10 & 11 & 55.22703 & 0.21765 & 348.718 & 86.1 & $2.0 \times 10^{-3}$ \\
\hline 15 & 17 & 18 & 55.72139 & 0.36511 & 0.136 & 86.7 & $2.2 \times 10^{4}$ \\
\hline 16 & 20 & 21 & 60.26429 & 0.30095 & 0.489 & 90.8 & $1.5 \times 10^{3}$ \\
\hline 17 & 23 & 24 & 62.72567 & 0.22736 & 1.114 & 92.3 & $2.2 \times 10^{2}$ \\
\hline 18 & 12 & 13 & 66.00061 & 0.21399 & 527.987 & 93.1 & $1.0 \times 10^{-3}$ \\
\hline 19 & 16 & 17 & 66.06384 & 0.39413 & 0.007 & 93.1 & $9.6 \times 10^{6}$ \\
\hline 20 & 19 & 20 & 72.22071 & 0.35381 & 0.011 & 90.7 & $3.4 \times 10^{6}$ \\
\hline 21 & 15 & 16 & 76.76666 & 0.32585 & 0.554 & 85.7 & $1.2 \times 10^{3}$ \\
\hline 22 & 22 & 23 & 76.82086 & 0.27437 & 0.049 & 85.6 & $1.3 \times 10^{5}$ \\
\hline 23 & 14 & 15 & 78.05113 & 0.42736 & 0.356 & 83.9 & $3.6 \times 10^{3}$ \\
\hline 24 & 18 & 19 & 84.85690 & 0.29359 & 2.008 & 67.8 & 63.3 \\
\hline 25 & 17 & 18 & 88.41772 & 0.44951 & 0.592 & 56.1 & $9.2 \times 10^{2}$ \\
\hline 26 & 21 & 22 & 93.40637 & 0.27267 & 5.150 & 35.0 & 4.6 \\
\hline 27 & 13 & 14 & 93.73493 & 0.34870 & 0.037 & 33.5 & $1.1 \times 10^{5}$ \\
\hline
\end{tabular}

${ }^{a}$ Errors, due to the finite number of decimal figures taken for the field, are estimated to be smaller than $1 \mathrm{kHz}$.

$$
P\left(\left|\widetilde{J}, K_{a}, K_{c}, M\right\rangle_{i}\right)=\sum_{n} P\left(\left|J, K_{a}, K_{c}, M\right\rangle_{n}\right) c_{n}^{i}\left(t_{f}\right) c_{n}^{i *}\left(t_{f}\right)
$$

where the sum runs over every initial field-free wave function that at time $t_{f}\left(t_{f}=v / x_{f}\right.$, with $x_{f}$ the position inside the plates where the field reaches its maximum value) contains some contribution of the $i$ Stark state. An accurate calculation of the populations in the field requires therefore solving the time-dependent Schrödinger equation for all the populated states outside the field. This is a huge problem for iodobenzene due to its small rotational constant. Even at $5 \mathrm{~K}$, there are around 1400 field-free states (taking into account the different $M$ 's) with a relative population larger than $10 \%$ of the maximum population. In principle, these states should be propagated to calculate the population distribution in the field. However, as we have seen above, our numerical calculation indicates that the time evolution is such that every avoided crossing is isolated from the others, involving only one pair of Stark states. Under these circumstances, a transition between two Stark states will not occur at an avoided crossing if the following condition is fulfilled [15]:

$$
\left|\left\langle\widetilde{J}, K_{a}, K_{c}, M\left|\frac{d \varepsilon}{d t} \frac{\partial}{\partial \varepsilon}\right| \widetilde{J}^{\prime}, K_{a}^{\prime}, K_{c}^{\prime}, M\right\rangle\right| \ll \Delta E,
$$

where $\Delta E$ is the energy difference between the two Stark states at the AC. Using the Hellmann-Feynman theorem and after some manipulation, this condition can be rewritten as

$$
\frac{\left|v(d \varepsilon / d x)\left\langle\widetilde{J}, K_{a}, K_{c}, M|-\mu \cos \theta| \widetilde{J}^{\prime}, K_{a}^{\prime}, K_{c}^{\prime}, M\right\rangle\right|}{(\Delta E)^{2}} \ll 1 .
$$

If $v$ is in $\mathrm{m} / \mathrm{s}, d \varepsilon / d x$ in $\mathrm{kV} / \mathrm{cm}^{2}$, the coupling term in $\mathrm{D}$, and $\Delta E$ in $\mathrm{MHz}$, Eq. (3.19) must be multiplied by the conversion factor 0.00801586 to have correct units.

Calculation of Eq. (3.19) is not trivial because the energy difference $\Delta E$ is very sensitive to the electric-field value for nearly degenerate Stark states. Therefore, the AC must be located with high precission. Nonetheless, this procedure is less time consuming than the numerical integration of the Schrödinger equation. Values for Eq. (3.19) and the various factors in it, at the ACs indicated in Figs. 2 and 3, are given 


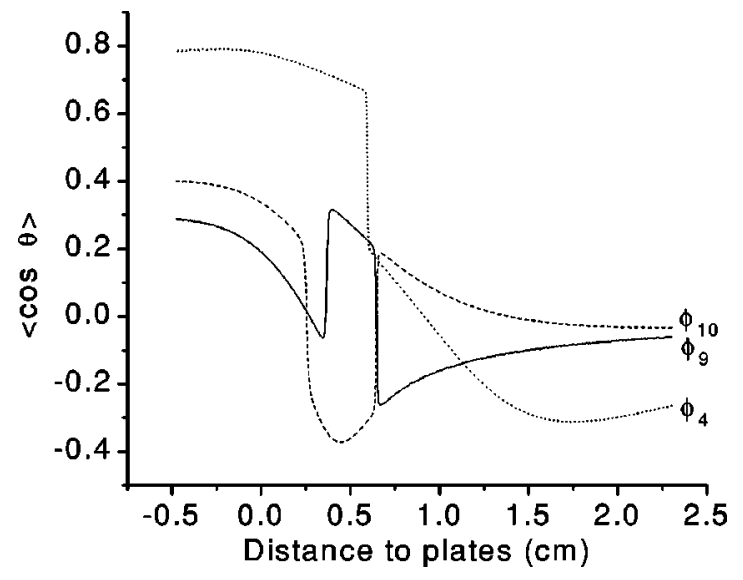

FIG. 4. Time evolution for three Stark states $\left(\phi_{4} \equiv|\widetilde{3}, 0,3,0\rangle\right.$, $\phi_{9} \equiv|\widetilde{6}, 0,6,0\rangle$, and $\left.\phi_{10} \equiv|\widetilde{4}, 2,2,0\rangle\right)$ of the orientation $\langle\cos \theta\rangle$, as a function of the distance to the edges of the conducting plates for $V_{0}=100 \mathrm{kV}$ and $a=1 \mathrm{~cm}$. The curves are constructed by plotting a point every $40 \mathrm{~ns}$ during the corresponding time evolution.

in Table I. Comparison with the results obtained from the numerical propagation of wave packets shows an excellent agreement, and it gives clues for explaining the behavior of wave functions at avoided crossings. Thus, narrow ACs are usually transversed diabatically even when the diabatic coupling between Stark states is very small (AC No. 1). Based on these facts, the calculation of the population distribution can be simplified by first determining, from Eq. (3.19), the ACs that would give rise to fully adiabatic or fully diabatic time evolution. Initial states which go through this kind of crossings do not need to be propagated. Only the wave functions which experience intermediate ACs need be propagated.

\section{Time evolution of the orientation}

Effects due to the presence of avoided crossings are not limited to changes in the population distribution of the molecular ensemble in the field. It is especially interesting to study the changes in orientation that take place due to the presence of avoided crossings. Figures 4 and 5 show the time evolution of $\langle\cos \theta\rangle$ ("the orientation") for several initial rotational states. The calculation of $\langle\cos \theta\rangle$ can be easily done, taking into account that

$$
\cos \theta=\frac{H_{\mathrm{rot}}-H}{\mu \varepsilon},
$$

since a wave function at time $t$ can be expanded either in the basis set of eigenstates of $H_{\text {rot }}$ or in the basis set of instantaneous eigenstates of $H(t)$ :

$$
\psi(t)=\sum_{n} c_{n}\left|J, K_{a}, K_{c}, M\right\rangle_{n} \equiv \sum_{n} d_{n}\left|\widetilde{J}, K_{a}, K_{c}, M\right\rangle_{n}
$$

Thus, the orientation at time $t$ is

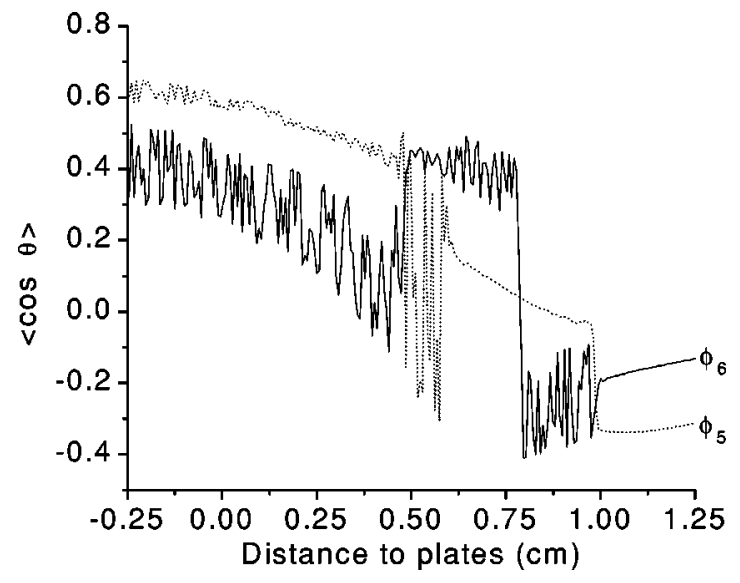

FIG. 5. Time evolution of the orientation for $\phi_{5} \equiv|\widetilde{4}, 0,4,0\rangle$ and $\phi_{6} \equiv|\widetilde{5}, 0,5,0\rangle$ under the same electric field as in Fig. 4. Note that the scale is different from the one employed in Fig. 4.

$$
\langle\psi(t)|\cos \theta| \psi(t)\rangle=1 /[\mu \varepsilon(t)] \sum_{n}\left(E_{\mathrm{rot}}^{n} c_{n}^{*} c_{n}-E_{S}^{n} d_{n}^{*} d_{n}\right),
$$

where $E_{\text {rot }}^{n}$ is the energy of the asymmetric-top eigenstate $n$, and $E_{S}^{n}$ is the energy of the Stark eigenstate $n$.

Only the lowest states reach a significant orientation at the electric field employed, but two interesting phenomena can be observed in Figs. 4 and 5.

(i) Flips of orientation. They take place when a crossing is transversed adiabatically, as can be seen for the three states in Fig. 4. For example $\langle\cos \theta\rangle$ increases gradually for $\phi_{10}$ until it hits AC No. 6, which is adiabatically transversed. At this point, the orientation decreases suddenly from 0.19 to -0.29 , and then, it keeps decreasing until AC No. 14 is reached. These flips are due to the usual interchange of character that occurs between two wave functions at isolated avoided crossing. This implies that wave functions whose time evolution is adiabatic experience a large change in character at the avoided crossing (the overlap integral between the wave function before and after the AC is small). On the other hand, if the avoided crossing is transversed diabatically, the orientation would evolve smoothly.

(ii) Irregular oscillations of orientation. In the intermediate case, i.e., when the wave function at a crossing becomes a coherent superposition of two or more instantaneous Stark states (a nonstationary state of the molecule-field Hamiltonian), the orientation oscillates in an irregular fashion as shown for the two states in Fig. 5. If the crossings that a molecule, initially described by the field-free state $\phi_{5}$ in Fig. 2 , experiences were transversed diabatically, it would end with $\langle\cos \theta\rangle=1$ for a high enough field. However, the wave function becomes a superposition of two Stark states [see Eq. (3.1)], giving rise to beats in the orientation, which are maintained after the maximum field is reached. These beats are stronger for $\phi_{6}$, which consists of a superposition of three Stark states. This phenomenon also implies that the creation of some specific pendular states is not possible with the brute force technique, unless the coherence established between several Stark states is broken by collisions. 


\section{SUMMARY AND DISCUSSION}

The properties of rotational wave packets of polar molecules created by the interaction of a permanent dipole moment with a static electric field have been well characterized by Friedrich and Herschbach [2]. The transition from fieldfree rotational eigenstates to pendular states is a dynamic process whose interpretation requires the solution of the time-dependent Schrödinger equation. This problem was studied for electric fields created by nonresonant laser pulses in Ref. [4]. The high intensities and short times involved in such processes allow nonadiabatic time evolution even in the absence of avoided crossings between instantanous molecule-field eigenstates (Floquet states). The case of a static electric field had not been treated until the present work because of the long propagations that are required. Besides, at the moderate field strengths involved, linear and symmetric-top molecules, in the vibronic ground state, usually have a simple energy level structure that does not allow nonadiabatic behavior. However, asymmetric tops can present numerous avoided crossings, which can give rise to nonadiabatic time evolution.

The main implications of our calculations are the following.

(i) We have identified states that evolve fully adiabatically, others that evolve fully diabatically, and a few states that evolve to coherent superpositions of several pendular states (intermediate behavior at ACs). Therefore, populations in the field cannot be calculated either from field-free populations or assuming complete mixing by the field.

(ii) Since the investigated avoided crossings are isolated, transition probabilities can be estimated from a simple expression containing the diabatic coupling matrix element, the energy gap between the two involved instantaneous Stark states, and the change rate of the field as a function of space coordinates and molecular velocity.

(iii) The orientation for wave packets consisting of superpositions of pendular states oscillates in time, even after the final static field is reached. This implies also that a few spe- cific pendular states cannot be created by brute force methods, unless the molecular velocity and/or the electric field variation can be controlled. This problem could be overcome because there is some flexibility in the design of the experimental configuration to obtain different fringe fields and since velocities can be modified with new methods $[16,17]$.

The method developed by Meijer and co-workers [16] is based on the fact that molecules in a low-field seeking state lose kinetic energy when entering an electric field. This velocity is not regained if the field is rapidly switched off. Therefore, succesive electric-field stages can slow down molecules. The method by Maddi et al. [17] is also based on the use of time-vaying electric-field gradients, but molecules in high-field seeking states or ground state atoms are used. The field, which is off when the molecules enter the field, is rapidly switched on when they are between the plates. Therefore, when molecules (or atoms) exit the plates transverse a field gradient and are slowed down. However, if avoided crossings exist as a function of field strength the dynamics can become more complex. For example, in the method by Meijer and co-workers, avoided crossings could be transversed adiabatically for molecules entering the field and diabatically when the field is switched off, since the timedependent perturbation acts much faster in this case. Thus, after one electric-field stage a molecule could end up in an internal state different from the initial state. In some cases, this state could be a high-field seeking state, and therefore the second electric-field stage would give rise to an increase in kinetic energy. We believe that studies like the one presented here can be relevant for the optimization of such schemes for the case of molecules in internal states that exhibit avoided crossings.

\section{ACKNOWLEDGMENTS}

This work was supported in part by the Spanish DGES (Project No. PB96-0881). We are grateful to Gerald Fraser for carefully reading the manuscript.
[1] H.J. Loesch and A. Remscheid, J. Chem. Phys. 93, 4779 (1990).

[2] B. Friedrich and D.R. Herschbach, Z. Phys. D: At., Mol. Clusters 18, 153 (1991); J. Phys. Chem. 95, 8118 (1991); Nature (London) 353, 412 (1991).

[3] B. Friedrich and D. Herschbach, Phys. Rev. Lett. 74, 4623 (1995).

[4] J. Ortigoso, M. Rodríguez, M. Gupta, and B. Friedrich, J. Chem. Phys. 110, 3870 (1999).

[5] D.T. Moore, L. Oudejans, and R.E. Miller, J. Chem. Phys. 110, 197 (1999).

[6] J.J. Larsen, H. Sakai, C.P. Safvan, I. Wendt-Larsen, and H. Stapelfeldt, J. Chem. Phys. 111, 7774 (1999).

[7] J. Bulthuis, J. Möller, and H.J. Loesch, J. Phys. Chem. A 101, 7684 (1997).

[8] P. Schwendner, C. Beck, and R. Schinke, Phys. Rev. A 58,
2203 (1998).

[9] J.A. Fleck, J.R. Morris, and M.D. Feit, Appl. Phys. 10, 129 (1976).

[10] C. DiLauro and I.M. Mills, J. Mol. Spectrosc. 21, 386 (1966).

[11] P.R. Bunker, Molecular Symmetry and Spectroscopy (Academic, New York, 1979).

[12] C.H. Townes and A.L. Schawlow, Microwave Spectroscopy (Dover, New York, 1975).

[13] A.M. Mirri and W. Caminati, Chem. Phys. Lett. 8, 409 (1971).

[14] J. Ortigoso, G.T. Fraser, and B.H. Pate, Phys. Rev. Lett. 82, 2856 (1999).

[15] T.-S. Ho and S.-I. Chu, Chem. Phys. Lett. 141, 315 (1987).

[16] H.L. Bethlem, G. Berden, and G. Meijer, Phys. Rev. Lett. 83, 1558 (1999).

[17] J.A. Maddi, T.P. Dinnen, and H. Gould, Phys. Rev. A 60, 3882 (1999). 